\title{
The surgical treatment of tumors of the fourth ventricle: a single-institution experience
}

\author{
Sherise D. Ferguson, MD, Nicholas B. Levine, MD, Dima Suki, PhD, Andrew J. Tsung, MD, \\ Fredrick F. Lang, MD, Raymond Sawaya, MD, Jeffrey S. Weinberg, MD, and \\ Ian E. McCutcheon, MD, FRCS(C)
}

Department of Neurosurgery, The University of Texas MD Anderson Cancer Center, Houston, Texas

OBJECTIVE Fourth ventricle tumors are rare, and surgical series are typically small, comprising a single pathology, or focused exclusively on pediatric populations. This study investigated surgical outcome and complications following fourth ventricle tumor resection in a diverse patient population. This is the largest cohort of fourth ventricle tumors described in the literature to date.

METHODS This is an 18-year (1993-2010) retrospective review of 55 cases involving patients undergoing surgery for tumors of the fourth ventricle. Data included patient demographic characteristics, pathological and radiographic tumor characteristics, and surgical factors (approach, surgical adjuncts, extent of resection, etc.). The neurological and medical complications following resection were collected and outcomes at 30 days, 90 days, 6 months, and 1 year were reviewed to determine patient recovery. Patient, tumor, and surgical factors were analyzed to determine factors associated with the frequently encountered postoperative neurological complications.

RESULTS There were no postoperative deaths. Gross-total resection was achieved in $75 \%$ of cases. Forty-five percent of patients experienced at least 1 major neurological complication, while $31 \%$ had minor complications only. New or worsening gait/focal motor disturbance $(56 \%)$, speech/swallowing deficits $(38 \%)$, and cranial nerve deficits $(31 \%)$ were the most common neurological deficits in the immediate postoperative period. Of these, cranial nerve deficits were the least likely to resolve at follow-up. Multivariate analysis showed that patients undergoing a transvermian approach had a higher incidence of postoperative cranial nerve deficits, gait disturbance, and speech/swallowing deficits than those treated with a telovelar approach. The use of surgical adjuncts (intraoperative navigation, neurophysiological monitoring) did not significantly affect neurological outcome. Twenty-two percent of patients required postoperative CSF diversion following tumor resection. Patients who required intraoperative ventriculostomy, those undergoing a transvermian approach, and pediatric patients (< 18 years old) were all more likely to require postoperative CSF diversion. Twenty percent of patients suffered at least 1 medical complication following tumor resection. Most complications were respiratory, with the most common being postoperative respiratory failure (14\%), followed by pneumonia (13\%).

CONCLUSIONS The occurrence of complications after fourth ventricle tumor surgery is not rare. Postoperative neurological sequelae were frequent, but a substantial number of patients had neurological improvement at long-term followup. Of the neurological complications analyzed, postoperative cranial nerve deficits were the least likely to completely resolve at follow-up. Of all the patient, tumor, and surgical variables included in the analysis, surgical approach had the most significant impact on neurological morbidity, with the telovelar approach being associated with less morbidity.

https://thejns.org/doi/abs/10.3171/2016.11.JNS161167

KEY WORDS fourth ventricle; tumor; telovelar approach; transvermian approach; oncology

I NTRAVENTRICULAR lesions represent a formidable surgical challenge. Surgery for intraventricular tumors is plagued by potential pitfalls due to proximity to deep eloquent structures and the risk of injury to perforating arteries supplying subcortical regions. Fourth ventricle tu- mors, in particular, present a unique surgical challenge because of adjacent cranial nerve nuclei in the ventricle floor and the risk of injury to cerebellar peduncles and dentate nuclei in the ventricle roof. Additionally, each wall of the fourth ventricle has critical vascular relationships. Tumors

ABBREVIATIONS CN = cranial nerve; EVD = extraventricular drain; KPS = Karnofsky Performance Status; MDACC = MD Anderson Cancer Center;

SUBMITTED May 16, 2016. ACCEPTED November 7, 2016.

INCLUDE WHEN CITING Published online April 14, 2017; DOI: 10.3171/2016.11.JNS161167. 
TABLE 1. Patient and tumor characteristics in 55 cases

\begin{tabular}{|c|c|}
\hline Characteristic & No. of Pts (\%) \\
\hline \multicolumn{2}{|l|}{ Sex } \\
\hline Female & $24(44)$ \\
\hline Male & $31(56)$ \\
\hline \multicolumn{2}{|l|}{ Age } \\
\hline Adult & $39(71)$ \\
\hline Child (<18 yrs) & $16(29)$ \\
\hline \multicolumn{2}{|l|}{ Presenting symptom* } \\
\hline Headache & $25(45)$ \\
\hline Nausea \& vomiting & $21(38)$ \\
\hline Unsteady gait & $18(33)$ \\
\hline Visual impairment & $16(29)$ \\
\hline Dizziness & $13(24)$ \\
\hline Cranial nerve deficit & $9(16)$ \\
\hline Weakness & $7(13)$ \\
\hline Memory impairment & $6(11)$ \\
\hline Sensory changes & $4(7)$ \\
\hline Seizure & $2(4)$ \\
\hline Altered mental status & $1(2)$ \\
\hline Speech problems & $4(7)$ \\
\hline \multicolumn{2}{|l|}{ Tumor pathology } \\
\hline Ependymoma & $20(36)$ \\
\hline Pilocytic astrocytoma & $6(11)$ \\
\hline Medulloblastoma & $5(9)$ \\
\hline Subependymoma & $5(9)$ \\
\hline Choroid plexus papilloma & $5(9)$ \\
\hline Hemangioblastoma & $4(7)$ \\
\hline Metastases & $5(9)$ \\
\hline Other & $5(9)$ \\
\hline Leptomeningeal disease & $2(4)$ \\
\hline \multicolumn{2}{|l|}{ Tumor growth characteristics* } \\
\hline Extension beyond 4th ventricle & $45(82)$ \\
\hline Lateral extension & $25(45)$ \\
\hline Anterior extension & $18(33)$ \\
\hline Caudal extension & $15(27)$ \\
\hline Extension in multiple directions & $19(35)$ \\
\hline No extension beyond 4 th ventricle & $10(18)$ \\
\hline
\end{tabular}

of the fourth ventricle may present with signs and symptoms of increased intracranial pressure resulting from hydrocephalus (headache, nausea/vomiting, vertigo, diplopia, papilledema, etc.) or from direct mass effect on the cerebellar hemispheres, vermis, or brainstem (e.g., ataxia, gait abnormalities, dysmetria, and long-tract abnormalities). Access to these tumors is typically accomplished via a suboccipital craniotomy/craniectomy followed by a transvermian or telovelar approach. The merits and limitations of each approach are thoroughly discussed in the literature, but no head-to-head comparison has hitherto been done. ${ }^{4,17,22,23}$
Intraventricular tumors represent $2 \%$ of intracranial lesions, and lesions of the fourth ventricle make up only a fraction of this subset. ${ }^{2}$ The overall paucity of cases thus limits data on clinical experience with the spectrum of pathologies associated in this region. The purpose of this paper is to review our experience with fourth ventricle tumors with an emphasis on short-term, approach-specific surgical complications and outcomes. This is the largest cohort of fourth ventricle tumor patients described in the literature to date.

\section{Methods}

This study was conducted under the auspices of an institutional review board-approved protocol. Consecutive patients $(n=55)$ who underwent resection of fourth ventricle tumors at The University of Texas MD Anderson Cancer Center (MDACC) between January 1, 1993, and September 30, 2010, were identified from the Brain and Spine Center Database. Clinical (i.e., surgical approach, postoperative outcomes) and pathological information was retrieved from this prospective database. Radiographic studies and reports were reviewed for tumor features. Tumors were classified based on whether they were contained completely within the fourth ventricle or displayed extension: caudally (into the foramen magnum), laterally (into the foramen of Luschka or cerebellopontine angle), or anteriorly (invading or distorting the brainstem). Data regarding surgical approach were obtained from detailed operative reports. The selection of surgical approach was based on surgeon preference and not a predetermined algorithm. The specifics governing each individual decision were not available for data collection. Extent of resection was determined by review of the postoperative MRI studies. Gross-total resection was defined as complete tumor resection with no evidence of residual tumor on postoperative MRI.

Postoperative neurological outcomes were reviewed using in-patient hospital records and outpatient clinic notes. Both new and worsening deficits were counted as complications. Immediate postoperative neurological function was assessed prior to discharge, and secondary follow-up was done on postoperative Days 30 and 90 and at 6 months and 1 year after surgery. Predictors of neurological outcome were analyzed using chi-square analyses to examine the significance of relationships between the variables ( $\mathrm{p}$ $<0.05$ was considered significant).

\section{Results}

\section{Patient and Tumor Characteristics}

Table 1 displays the characteristics of the 55 surgical patients. There was a slight male preponderance $(56 \%)$. The overall mean age was 35.5 years. The majority of patients were adults $(71 \%)$. The most common presenting symptoms were headaches $(45 \%)$, nausea and vomiting (38\%), gait difficulties (33\%), and visual changes (29\%). Only 9 patients $(16 \%)$ had cranial nerve deficits at initial presentation. Several patients presented with multiple symptoms.

The most common tumor types were ependymoma 
TABLE 2. Surgical factors and adjuncts

\begin{tabular}{|c|c|}
\hline Variable & No. of Pts (\%) \\
\hline \multicolumn{2}{|l|}{ Extent of resection } \\
\hline GTR & $41(75)$ \\
\hline STR & $13(24)$ \\
\hline Biopsy & $1(2)$ \\
\hline \multicolumn{2}{|l|}{ Surgical exposure } \\
\hline Craniotomy & $44(80)$ \\
\hline Craniectomy & $11(20)$ \\
\hline \multicolumn{2}{|l|}{ Surgical approach* } \\
\hline Transvermian & $24(44)$ \\
\hline Telovelar & $26(48)$ \\
\hline Other & $4(7)$ \\
\hline \multicolumn{2}{|l|}{ Surgical adjuncts } \\
\hline Intraop EVD & $14(25)$ \\
\hline Intraop monitoring & $18(33)$ \\
\hline Intraop navigation & $49(89)$ \\
\hline Ultrasound only & $15(27)$ \\
\hline MRI only & $1(2)$ \\
\hline Ultrasound \& MRI & $33(60)$ \\
\hline Preop CSF diversion & $9(16)$ \\
\hline Postop CSF diversion & $12(22)$ \\
\hline
\end{tabular}

GTR = gross-total resection; STR = subtotal resection .

* Data available for 54 cases only.

$(36 \%)$ and pilocytic astrocytoma (11\%). Fewer than $10 \%$ of lesions were metastases from tumors outside the brain. Cases listed as "other" included 3 cysts (2 dermoid cysts and 1 neuroepithelial cyst) and 1 case each of lymphoma and anaplastic astrocytoma. Only $4 \%$ of patients had confirmed leptomeningeal disease at the time of surgery. Most tumors (82\%) extended beyond the confines of the fourth ventricle. Lateral extension (particularly into the foramen of Luschka) was the most frequently observed extension, occurring in $45 \%$ of cases, while caudal extension into the foramen of Magendie was seen less often.
Anterior extension, resulting in invagination or invasion of the brainstem, was apparent in one-third of cases.

\section{Surgical Approach and Extent of Resection}

In all but 1 case, in which an open biopsy was performed, complete resection was the goal of surgery. MDACC is a tertiary referral center, and 16 patients in this series (29\%) were undergoing repeat operations for recurrent tumor or following previous subtotal resections. All lesions were approached through a standard midline or paramedian posterior incision. The posterior fossa was exposed using a craniotomy in $80 \%$ of cases; in the remaining cases craniectomy was performed. The majority of patients (70\%) were placed in the lateral position; specific positioning data were missing for 2 cases. A unilateral telovelar approach was used in $48 \%$ of cases, and in most others the tumor was approached by splitting the vermis. In 4 cases (listed as "other" in Table 2) the surgical approach could not be definitively classified because these were repeat operations in which the previous surgical cavity was reentered for tumor exposure and resection. We did not find a statistically significant relationship between the surgical approach chosen and tumor anatomical characteristics (e.g., lateral or caudal extension). We also did not find a relationship between tumor histology and surgical approach $\left(\chi^{2}[14]=11.70, p=0.63\right)$. However, the telovelar approach was used more commonly in adult patients. A transvermian approach was used in $11(69 \%)$ of the 16 pediatric cases, and a telovelar approach in only $2(12 \%)$; in contrast, a telovelar approach was used in $24(63 \%)$ of the 38 cases involving adults $\left(\chi^{2}[2]=12.97, \mathrm{p}=0.002\right)$. The transvermian approach was used more frequently for recurrent tumor/repeat operations. Specifically, $50 \%$ of repeat operations were done with a transvermian approach versus only $31 \%$ with a telovelar approach, but this finding did not quite reach statistical significance $\left(\chi^{2}[2]=5.45\right.$, $\mathrm{p}=0.065)$. The relationship between surgeon experience and surgical approach is presented in Fig. 1; there was no statistically significant relationship noted $\left(\chi^{2}[6]=4.28, p\right.$ $=0.638$ ). Because this study includes a wide time span, we analyzed whether the surgical approach was influenced by

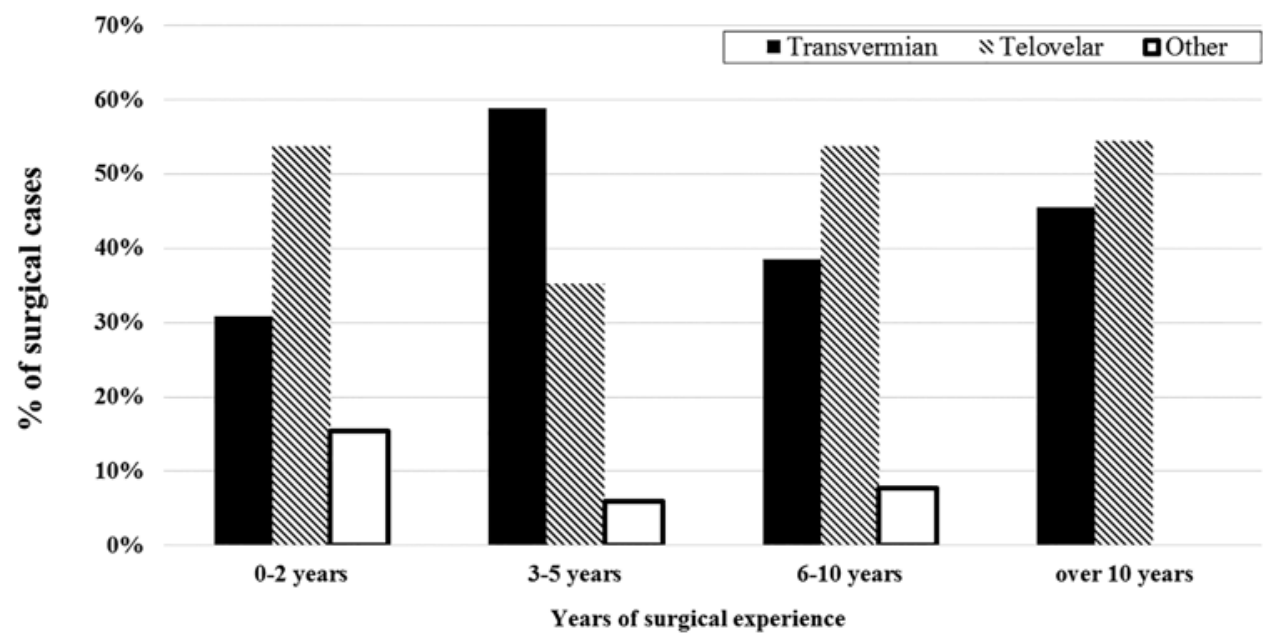

FIG. 1. Relationship between surgical experience and surgical approach. 


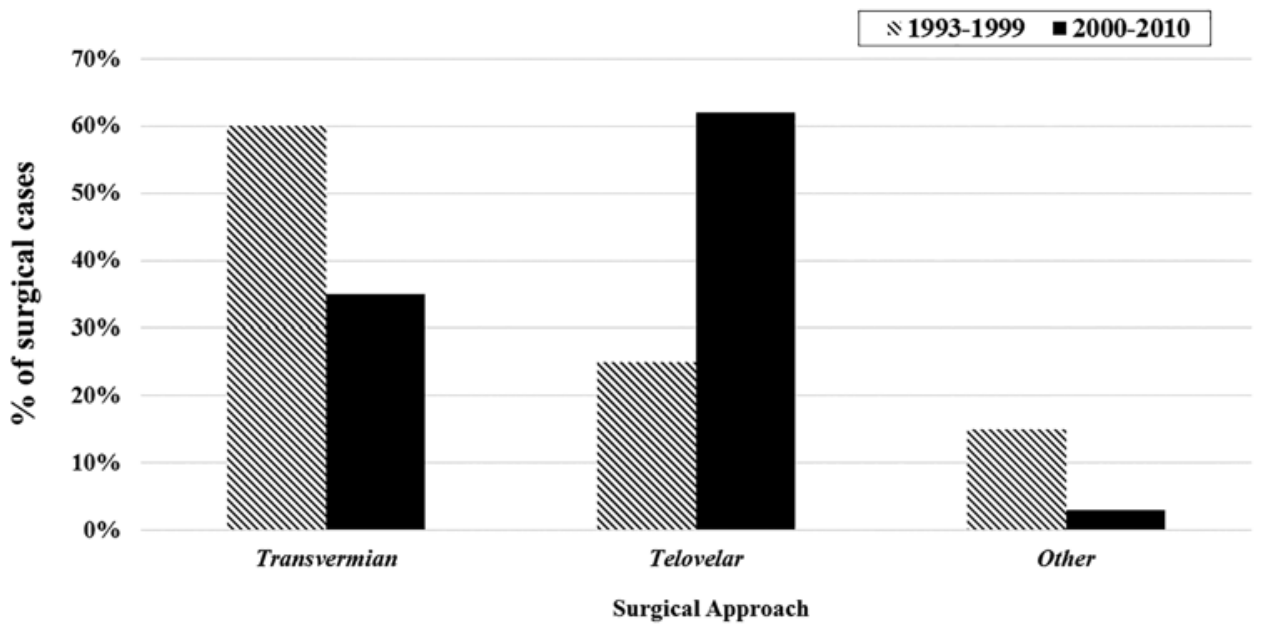

FIG. 2. Relationship between year of surgery and surgical approach.

the year of surgery (Fig. 2). Statistical analysis indicated that patients undergoing surgery between the years 2000 and 2010 were more likely to have a telovelar approach than patients who underwent surgery in the 1990s $\left(\chi^{2}\right.$ [2] $=7.74, p=0.021)$. Gross-total resection was achieved in $75 \%$ of cases. There was no statistically significant correlation between surgical approach and extent of resection $\left(\chi^{2}[2]=0.026, p=0.987\right)$. There was also no statistically significant correlation between tumor histology and extent of resection $\left(\chi^{2}[7]=9.766, p=0.202\right)$.

\section{Surgical Adjuncts}

Some form of intraoperative navigation was used in $89 \%$ of cases (Table 2). MRI-guided navigation was used for planning tumor exposure. Ultrasonography was typically applied following craniotomy/craniectomy for real-

TABLE 3. Neurological complications

\begin{tabular}{lc}
\hline \multicolumn{1}{c}{ Complication } & No. of Pts (\%) \\
\hline Postop neurological complication & \\
\hline Yes & $42(76)$ \\
\hline No & $13(24)$ \\
\hline Major complication & $25(45)$ \\
\hline Minor complication & $17(31)$ \\
\hline Gait disturbance/motor deficit & $31(56)$ \\
\hline Speech/swallowing deficit & $21(38)$ \\
\hline Cranial nerve deficit & $17(31)$ \\
\hline Visual impairment & $16(29)$ \\
\hline Sensory deficit & $1(2)$ \\
\hline Postop hemorrhage & $1(2)$ \\
\hline Meningitis & $3(5)$ \\
\hline CSF leak & $4(7)$ \\
\hline Wound infection & $4(7)$ \\
\hline Bladder dysfunction & $0(0)$ \\
\hline Seizures & $0(0)$ \\
\hline Hydrocephalus & $6(11)$ \\
\hline Pneumocephalus & $2(4)$ \\
\hline
\end{tabular}

time imaging of tumor location, and again after resection to inspect the surgical cavity for residual tumor. In the majority of cases, frameless stereotactic image guidance was used in conjunction with intraoperative ultrasonography $(60 \% ; n=33)$. In only $33 \%$ was intraoperative neurophysiological monitoring done. Fourteen patients $(25 \%)$ had intraoperative CSF diversion in the form of an extraventricular drain (EVD), and an additional 16\% had preoperative CSF diversion.

\section{Postoperative Outcome}

There were no immediate postoperative deaths. Postoperative Karnofsky Performance Status (KPS) scores were documented in 55 cases. Compared with their preoperative function, 28 patients $(51 \%)$ had worsening of their KPS score immediately following surgery while only 3 $(5.5 \%)$ had improvement. In 24 patients (44\%) no change was reported.

\section{Neurological Morbidity}

Only one-quarter of cases were free of complications, and in $45 \%$ there were complications categorized as major (Table 3). Complications were defined as major if they significantly impacted patients' activities of daily living, altered the expected course of recovery, required an additional surgical procedure (e.g., wound washout, tracheotomy, etc.), significantly extended hospital stay, or required admission to inpatient rehabilitation for more than 2 weeks. New or worsening cranial nerve deficits were all categorized as major. Complications other than those described were regarded as minor. Our analysis found no statistically significant relationship between tumor histology and neurological outcome $\left(\chi^{2}[7]=8.554, \mathrm{p}=0.286\right.$. Additionally, bivariate analysis demonstrated no statistically significant relationship between individual surgeon experience and neurological outcome $\left(\chi^{2}[3]=2.91, \mathrm{p}=\right.$ 0.406).

\section{Gait and Focal Motor Deficits}

The most common postoperative complication was new or worsening gait/focal motor disturbance, which af- 
TABLE 4. Postoperative gait/focal motor deficit

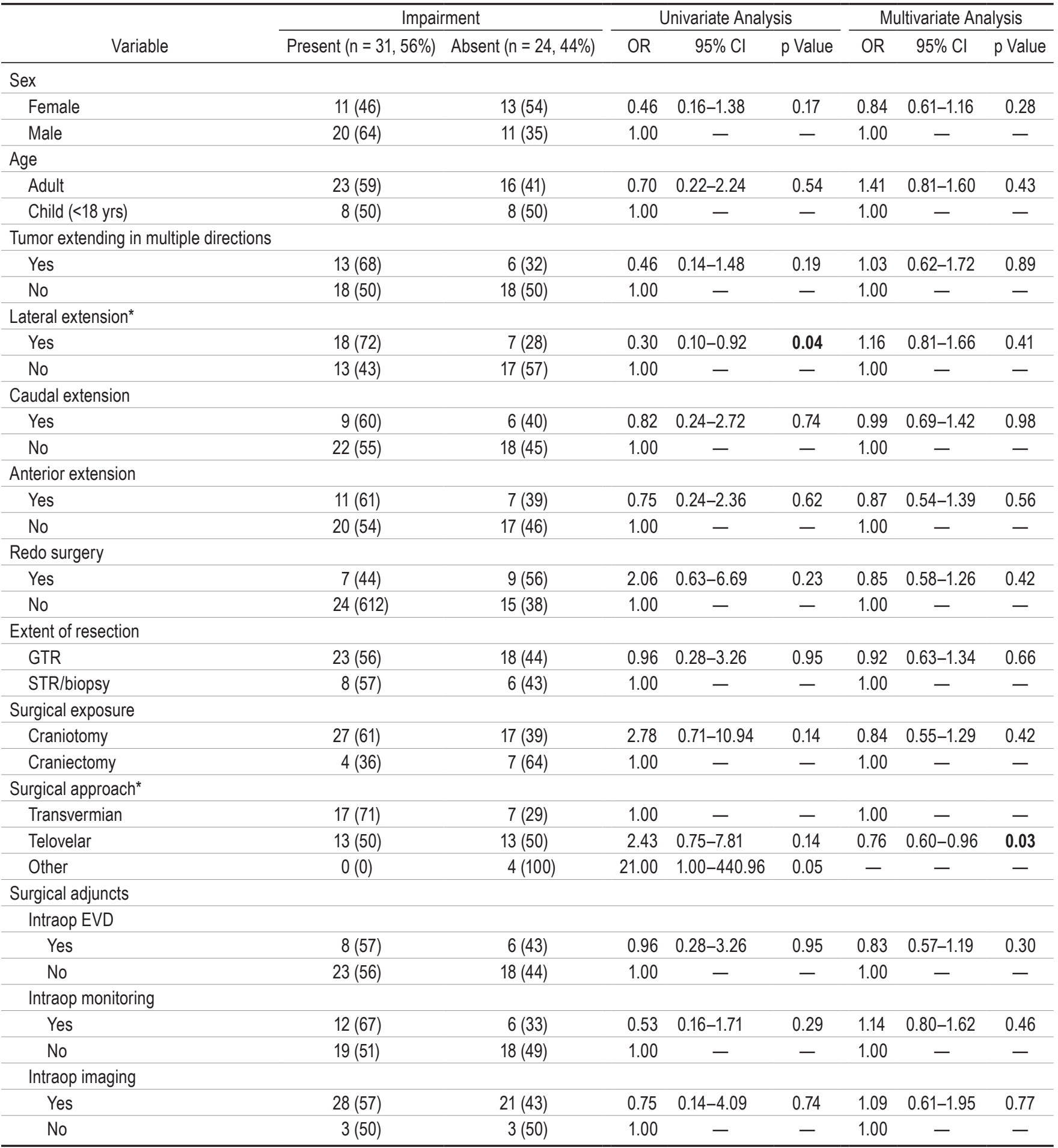

Data are presented as number of patients (\%). Boldface type indicates statistical significance.

${ }^{*} p \leq 0.05$.

fected 56\% ( $\mathrm{n}=31)$ of patients postoperatively (Tables 3 and 4). In univariate analysis we found that tumors with lateral extension were associated with increased incidence of postoperative gait disturbance ( $72 \%$ vs $43 \%, \mathrm{p}<0.05$ ). After controlling for other factors in a multivariate analy- sis, the only factor associated with postoperative gait/focal motor disturbance was use of the telovelar approach, after which deficits were less likely $(\mathrm{p}<0.05)$. Thirty-day recovery data were available for 25 of the 31 patients; $12.5 \%$ of these 25 patients had complete resolution of gait distur- 
bance after 30 days. Ninety-day follow-up was available for 21 of the 31 patients, and $25 \%$ of these 21 patients had symptom resolution at this time point. At 6 months and 1 year of follow-up, the rates of symptom resolution were $31 \%$ and $53 \%$, respectively.

\section{Speech/Swallowing Deficits}

Twenty-one patients (38\%) had worse or newly documented speech and/or swallowing difficulties following surgical intervention (Tables 3 and 5). Of these patients, 6 (29\%) required a tracheostomy and/or percutaneous feeding tube placement during their hospital admission. In a univariate analysis, we found that tumors demonstrating anterior extension (i.e., abutting or invading the brainstem) were significantly associated with postoperative swallowing/speech deficits. Specifically, $61 \%$ of cases with anterior extension were complicated by new or worsening speech/ swallowing deficits versus $27 \%$ of those without anterior extension $(p<0.05)$. There was no significant effect of caudal extension or lateral extension. Additionally, patients with tumor extending in multiple directions were significantly more likely to experience a postoperative speech/ swallowing deficit $(58 \%$ vs $28 \%, \mathrm{p}<0.05)$. The impact of anterior extension on speech/swallowing deficits remained significant in the multivariate analysis $(\mathrm{p}<0.05)$. Multivariate analysis also revealed that surgical approach significantly affected outcome, with the telovelar approach associated with fewer postoperative speech/swallowing deficits. Among patients undergoing a telovelar approach, $31 \%$ experienced postoperative speech/swallowing deficits compared with $54 \%$ of those who underwent a transvermian approach $(\mathrm{p}<0.05)$.

At 30-day follow-up, speech/swallowing deficits had resolved in half of the patients $(50 \%$; data missing for 3 cases). By 90-day follow-up $80 \%$ of patients reported complete resolution of speech/swallowing symptoms (data missing for 6 cases); this percentage increased to $83 \%$ at 6-month follow-up. At 1 year after surgery $100 \%$ of patients with available data $(\mathrm{n}=11)$ reported complete resolution.

\section{Cranial Nerve Deficits}

Following resection, 17 patients $(31 \%)$ had new or worsening cranial nerve palsies (Tables 3 and 6). In many of these cases $(70 \%)$, multiple cranial nerves were affected. Two patients each had 4 cranial nerve deficits; this was the maximum number of new cranial nerve deficits recorded in a single patient. The most common cranial nerve affected was cranial nerve (CN) VI, followed by $\mathrm{CN}$ VII. In a univariate analysis, both tumor extension in multiple dimensions and caudal tumor extension were associated with new or worsening cranial nerve deficits. Specifically, $58 \%$ of patients with tumor extension in multiple dimensions experienced postoperative cranial nerve deficits versus $17 \%$ of patients without $(\mathrm{p}<0.05)$, and $53 \%$ of patients with caudal extension experienced postoperative cranial nerve deficits versus $22 \%$ of patients without $(\mathrm{p}<0.05)$. Surgical approach also had a statistically significant impact on cranial nerve outcome $(\mathrm{p}<0.05)$. In a multivariate analysis, the only factor that significantly influenced cranial nerve outcome was the nature of the surgical ap- proach. The telovelar approach was associated with significantly less new/worsening postoperative cranial nerve deficits, with only $19 \%$ of patients being affected versus $50 \%$ of patients undergoing a transvermian approach $(\mathrm{p}$ $<0.05$ ). From the available follow-up data (data missing for 3 cases), we found that at 30-day follow-up, only $7 \%$ of patients had complete resolution of their cranial nerve deficits. This result did not substantially improve at 90day follow-up (8\%). However, at 1 year after surgery $30 \%$ of patients reported complete resolution of cranial nerve deficits (data missing for 7 cases for this time point).

\section{Other Surgical Complications}

The remaining neurological complications affected a smaller percentage of patients (Table 3). The occurrence of postoperative hemorrhage was a rare event, present in only 1 patient in this series. Infectious complications (meningitis, wound infection, etc.) were slightly more common. Three patients $(5 \%)$ developed meningitis, but in no case was it complicated by the development of cerebral abscess or encephalitis. Four patients $(7 \%)$ had CSF leaks. Two patients (4\%) suffered symptomatic pneumocephalus.

\section{Postoperative CSF Diversion}

Twelve patients $(22 \%)$ required postoperative CSF diversion following tumor resection (Table 7). In a multivariate analysis we found that pediatric patients were more likely to require CSF diversion after surgery; $37 \%$ of children were treated with CSF shunting following fourth ventricular tumor resection compared with $15 \%$ of adult patients $(\mathrm{p}<0.05)$. The likelihood of postoperative CSF diversion was not influenced by tumor pathology, tumor extension pattern, or extent of resection. Patients who underwent a telovelar approach were less likely to require postoperative CSF diversion (8\%) than those who underwent a transvermian approach $(42 \%)(\mathrm{p}<0.05)$. Among patients who had intraoperative EVD placement, $64 \%$ eventually required a permanent shunt, making this also a factor significantly associated with postoperative CSF diversion in the multivariate analysis $(\mathrm{p}<0.05)$.

\section{Medical Complications}

Eleven patients $(20 \%)$ suffered at least 1 medical complication following tumor resection; 9 (16\%) experienced major complications and 2 (4\%) had minor complications. The most common medical complications were respiratory in nature, including respiratory failure (requiring prolonged intubation) in $14 \%$ of patients and pneumonia in $13 \%$. Three patients had gastrointestinal bleeding, and 2 patients suffered deep vein thrombosis. There were no cardiac complications in this series.

\section{Discussion}

In the literature, it is accepted that resection of tumors of the fourth ventricle can be challenging due to adjacent critical structures; however, surgical complications have rarely been reviewed in detail. In addition, many studies focus on a specific pathology, such as medulloblastoma or ependymoma, or are limited to pediatric populations. ${ }^{2}$ This is the largest series of cases involving fourth ventricle 
TABLE 5. Postoperative speech/swallowing deficits

\begin{tabular}{|c|c|c|c|c|c|c|c|c|}
\hline \multirow{2}{*}{ Variable } & \multicolumn{2}{|c|}{ Impairment } & \multicolumn{3}{|c|}{ Univariate Analysis } & \multicolumn{3}{|c|}{ Multivariate Analysis } \\
\hline & Present $(n=21,38 \%)$ & Absent $(n=34,62 \%)$ & OR & $95 \% \mathrm{Cl}$ & p Value & OR & $95 \% \mathrm{Cl}$ & p Value \\
\hline \multicolumn{9}{|l|}{ Sex } \\
\hline Female & $9(37)$ & $15(62)$ & 0.95 & $0.32-2.85$ & 0.93 & 0.98 & $0.72-1.33$ & 0.89 \\
\hline Male & $12(39)$ & $19(61)$ & 1.00 & - & - & 1.00 & - & - \\
\hline \multicolumn{9}{|l|}{ Age } \\
\hline Adult & $16(41)$ & $23(59)$ & 0.65 & $0.19-2.25$ & 0.50 & 1.11 & $0.81-1.53$ & 0.49 \\
\hline Child (<18 yrs) & $5(31)$ & $11(69)$ & 1.00 & - & - & 1.00 & - & - \\
\hline \multicolumn{9}{|c|}{ Tumor extending in multiple directions* } \\
\hline Yes & $11(58)$ & $8(42)$ & 0.28 & $0.09-0.90$ & 0.03 & 0.95 & $0.59-1.53$ & 0.83 \\
\hline No & $10(28)$ & $26(72)$ & 1.00 & - & - & 1.00 & - & - \\
\hline \multicolumn{9}{|l|}{ Lateral extension } \\
\hline Yes & $12(48)$ & $13(52)$ & 0.46 & $0.15-1.40$ & 0.17 & 1.21 & $0.86-1.70$ & 0.26 \\
\hline No & $9(30)$ & $21(70)$ & 1.00 & - & - & 1.00 & - & - \\
\hline \multicolumn{9}{|l|}{ Caudal extension } \\
\hline Yes & $7(47)$ & $8(53)$ & 0.61 & $0.18-2.05$ & 0.43 & 1.08 & $0.77-1.51$ & 0.66 \\
\hline No & $14(35)$ & $26(65)$ & 1.00 & - & - & 1.00 & - & - \\
\hline \multicolumn{9}{|l|}{ Anterior extension* } \\
\hline Yes & $11(61)$ & $7(39)$ & 0.24 & $0.07-0.78$ & 0.02 & 1.54 & $0.99-2.40$ & 0.05 \\
\hline No & $10(27)$ & $27(73)$ & 1.00 & - & - & 1.00 & - & - \\
\hline \multicolumn{9}{|l|}{ Redo surgery } \\
\hline Yes & $5(31)$ & $11(69)$ & 1.53 & $0.44-5.26$ & 0.50 & 1.02 & $0.71-1.46$ & 0.93 \\
\hline No & $16(41)$ & $23(59)$ & 1.00 & - & - & 1.00 & - & - \\
\hline \multicolumn{9}{|l|}{ Extent of resection } \\
\hline GTR & $17(41)$ & $24(58)$ & 1.77 & $0.47-6.60$ & 0.39 & 0.78 & $0.55-1.12$ & 0.18 \\
\hline STR/biopsy & $4(29)$ & $10(71)$ & 1.00 & - & - & 1.00 & - & - \\
\hline \multicolumn{9}{|l|}{ Surgical exposure } \\
\hline Craniotomy & $19(43)$ & $25(57)$ & 3.42 & $0.66-17.70$ & 0.14 & 0.84 & $0.56-1.26$ & 0.40 \\
\hline Craniectomy & $2(18)$ & $9(82)$ & 1.00 & - & - & 1.00 & - & - \\
\hline \multicolumn{9}{|l|}{ Surgical approach* } \\
\hline Transvermian & $13(54)$ & $11(46)$ & 1.00 & - & - & 1.00 & - & - \\
\hline Telovelar & $8(31)$ & $18(69)$ & 2.66 & $0.84-8.45$ & 0.10 & 0.76 & $0.60-0.95$ & 0.02 \\
\hline Other & $0(0)$ & $4(100)$ & 10.56 & $0.51-217.75$ & 0.13 & - & - & - \\
\hline \multicolumn{9}{|l|}{ Surgical adjuncts } \\
\hline \multicolumn{9}{|l|}{ Intraop EVD } \\
\hline Yes & $6(43)$ & $8(57)$ & 0.77 & $0.22-2.64$ & 0.68 & 1.05 & $0.75-1.48$ & 0.75 \\
\hline No & $15(36)$ & $26(63)$ & 1.00 & - & - & 1.00 & - & - \\
\hline \multicolumn{9}{|l|}{ Intraop monitoring } \\
\hline Yes & $8(44)$ & $10(56)$ & 0.68 & $0.22-2.32$ & 0.51 & 1.14 & $0.82-1.58$ & 0.44 \\
\hline No & $13(35)$ & $24(65)$ & 1.00 & - & - & 1.00 & - & - \\
\hline \multicolumn{9}{|l|}{ Intraop imaging } \\
\hline Yes & $19(39)$ & $30(61)$ & 0.79 & $0.13-4.74$ & 0.80 & 1.08 & $0.62-1.87$ & 0.78 \\
\hline No & $2(33)$ & $4(67)$ & 1.00 & - & - & 1.00 & - & - \\
\hline
\end{tabular}

Data are presented as number of patients (\%). Boldface type indicates statistical significance.

${ }^{*} p \leq 0.05$.

tumors described and also the largest cohort $(n=39)$ of adult patients with fourth ventricle tumors reported. Other large series recently published include those reported by Han et al. ${ }^{8}$ (with 50 patients) and Tomasello et al. ${ }^{23}$ (with 45 patients); in both of these series, the telovelar approach was used exclusively, but neither study addressed compli- cations in detail. We found that the incidence of complications after resection of fourth ventricle tumors is not trivial. Although there was no perioperative death in our series, the majority of patients experienced at least 1 complication (either major or minor). A description of the pitfalls and complications is essential to provide realistic expectations 
TABLE 6. Postoperative cranial nerve deficits

\begin{tabular}{|c|c|c|c|c|c|c|c|c|}
\hline \multirow[b]{2}{*}{ Variable } & \multicolumn{2}{|c|}{ Impairment } & \multicolumn{3}{|c|}{ Univariate Analysis } & \multicolumn{3}{|c|}{ Multivariate Analysis } \\
\hline & Present $(n=17,31 \%)$ & Absent $(n=38,69 \%)$ & OR & $95 \% \mathrm{Cl}$ & $\mathrm{p}$ Value & OR & $95 \% \mathrm{Cl}$ & $p$ Value \\
\hline \multicolumn{9}{|l|}{ Sex } \\
\hline Female & $5(21)$ & $19(79)$ & 0.42 & $0.12-1.41$ & 0.16 & 0.85 & $0.66-1.10$ & 0.21 \\
\hline Male & $12(39)$ & $19(61)$ & 1.00 & - & - & 1.00 & - & - \\
\hline \multicolumn{9}{|l|}{ Age } \\
\hline Adult & $11(28)$ & $28(72)$ & 1.53 & $0.45-5.22$ & 0.50 & 0.89 & $0.68-1.16$ & 0.39 \\
\hline Child $(<18)$ & $6(37)$ & $10(62)$ & 1.00 & - & - & 1.00 & - & - \\
\hline \multicolumn{9}{|c|}{ Tumor extending in multiple directions* } \\
\hline Yes & $11(58)$ & $8(42)$ & 0.15 & $0.04-0.51$ & 0.03 & 1.36 & $0.91-2.03$ & 0.13 \\
\hline No & $6(17)$ & $30(83)$ & 1.00 & - & - & 1.00 & - & - \\
\hline \multicolumn{9}{|l|}{ Lateral extension } \\
\hline Yes & $11(44)$ & $14(56)$ & 0.32 & $0.10-1.05$ & 0.06 & 1.22 & $0.91-1.62$ & 0.17 \\
\hline No & $6(20)$ & $24(80)$ & 1.00 & - & - & 1.00 & - & - \\
\hline \multicolumn{9}{|l|}{ Caudal extension* } \\
\hline Yes & $8(53)$ & $7(47)$ & 0.25 & $0.07-0.89$ & 0.03 & 1.27 & $0.95,1.69$ & 0.10 \\
\hline No & $9(22)$ & $31(78)$ & 1.00 & - & - & 1.00 & - & - \\
\hline \multicolumn{9}{|l|}{ Anterior extension } \\
\hline Yes & $8(44)$ & $10(56)$ & 0.40 & $0.12-1.33$ & 0.14 & 1.07 & $0.74-1.56$ & 0.69 \\
\hline No & $9(24)$ & $28(76)$ & 1.00 & - & - & 1.00 & - & - \\
\hline \multicolumn{9}{|l|}{ Redo surgery } \\
\hline Yes & $3(19)$ & $13(81)$ & 2.43 & $0.59-10.00$ & 0.22 & 0.92 & $0.68-1.25$ & 0.59 \\
\hline No & $14(36)$ & $25(64)$ & 1.00 & - & - & 1.00 & - & - \\
\hline \multicolumn{9}{|l|}{ Extent of resection } \\
\hline GTR & $13(32)$ & $28(68)$ & 1.16 & $0.31-4.40$ & 0.83 & 0.97 & $0.72-1.31$ & 0.85 \\
\hline STR/biopsy & $4(29)$ & $10(71)$ & 1.00 & - & - & 1.00 & - & - \\
\hline \multicolumn{9}{|l|}{ Surgical exposure } \\
\hline Craniotomy & $16(36)$ & $28(64)$ & 5.71 & $0.67-48.83$ & 0.11 & 1.01 & $0.72-1.41$ & 0.97 \\
\hline Craniectomy & $1(9)$ & $10(91)$ & 1.00 & - & - & 1.00 & - & - \\
\hline \multicolumn{9}{|l|}{ Surgical approach* } \\
\hline Transvermian & $12(50)$ & $12(50)$ & 1.00 & - & - & 1.00 & - & - \\
\hline Telovelar & $5(19)$ & $21(81)$ & 4.20 & $1.19-14.83$ & 0.03 & 0.74 & $0.61-0.90$ & $<0.001$ \\
\hline Other & $0(0)$ & $4(100)$ & 9.00 & $0.44-185.37$ & 0.15 & - & - & - \\
\hline \multicolumn{9}{|l|}{ Surgical adjuncts } \\
\hline \multicolumn{9}{|l|}{ Intraop EVD } \\
\hline Yes & $6(43)$ & $8(57)$ & 0.49 & $0.14-1.73$ & 0.27 & 1.01 & $0.76-1.35$ & 0.92 \\
\hline No & $11(27)$ & $30(73)$ & 1.00 & - & - & 1.00 & - & - \\
\hline \multicolumn{9}{|l|}{ Intraop monitoring } \\
\hline Yes & $7(39)$ & $11(61)$ & 0.58 & $0.18-1.92$ & 0.37 & 1.02 & $0.77-1.35$ & 0.86 \\
\hline No & $10(27)$ & $27(73)$ & 1.00 & - & - & 1.00 & - & - \\
\hline \multicolumn{9}{|l|}{ Intraop imaging } \\
\hline Yes & $16(33)$ & $33(67)$ & 0.41 & $0.04-3.83$ & 0.44 & 1.52 & $0.95-2.41$ & 0.08 \\
\hline No & $1(17)$ & $5(83)$ & 1.00 & - & - & 1.00 & - & - \\
\hline
\end{tabular}

Data are presented as number of patients (\%). Boldface type indicates statistical significance.

${ }^{*} p \leq 0.05$.

regarding patient outcome and for appropriate counseling regarding postoperative course and recovery.

\section{Gait}

Among neurological complications, gait/focal motor disturbance predominated, affecting more than half of the patients (56\%). In light of the anatomical challenges, this result is not unexpected. The cerebellar peduncles converge on the lateral wall and roof of the fourth ventricle and may be damaged during resection. The inferior and 
TABLE 7. Postoperative CSF diversion

\begin{tabular}{|c|c|c|c|c|c|c|c|c|}
\hline \multirow[b]{2}{*}{ Variable } & \multicolumn{2}{|c|}{ Impairment } & \multicolumn{3}{|c|}{ Univariate Analysis } & \multicolumn{3}{|c|}{ Multivariate Analysis } \\
\hline & Present $(n=12,22 \%)$ & Absent $(n=43,78 \%)$ & OR & $95 \% \mathrm{Cl}$ & p Value & OR & $95 \% \mathrm{Cl}$ & p Value \\
\hline \multicolumn{9}{|l|}{ Sex } \\
\hline Female & $3(12)$ & $21(88)$ & 0.35 & $0.08-1.47$ & 0.15 & 0.91 & $0.75-1.12$ & 0.37 \\
\hline Male & $9(29)$ & $22(71)$ & 1.00 & - & - & 1.00 & - & - \\
\hline \multicolumn{9}{|l|}{ Age } \\
\hline Adult & $6(15)$ & $33(85)$ & 3.30 & $0.87-12.53$ & 0.08 & 0.80 & $0.65-0.99$ & 0.04 \\
\hline Child (<18 yrs) & $6(37)$ & $10(62)$ & 1.00 & - & - & 1.00 & - & - \\
\hline \multicolumn{9}{|c|}{ Tumor extending in multiple directions } \\
\hline Yes & $5(26)$ & $14(74)$ & 0.68 & $0.18-2.51$ & 0.56 & 1.17 & $0.85-1.59$ & 0.32 \\
\hline No & $7(19)$ & $29(81)$ & 1.00 & - & - & 1.00 & - & - \\
\hline \multicolumn{9}{|l|}{ Lateral extension } \\
\hline Yes & $6(24)$ & $19(76)$ & 0.79 & $0.22-2.85$ & 0.72 & 1.01 & $0.81-1.26$ & 0.92 \\
\hline No & $6(20)$ & $24(80)$ & 1.00 & - & - & 1.00 & - & - \\
\hline \multicolumn{9}{|l|}{ Caudal extension } \\
\hline Yes & $5(33)$ & $10(67)$ & 0.42 & $0.11-1.63$ & 0.21 & 1.14 & $0.91-1.42$ & 0.24 \\
\hline No & $7(17)$ & $33(82)$ & 1.00 & - & - & 1.00 & - & - \\
\hline \multicolumn{9}{|l|}{ Anterior extension } \\
\hline Yes & $2(11)$ & $16(89)$ & 2.96 & $0.57-15.26$ & 0.19 & 0.81 & $0.60-1.08$ & 0.14 \\
\hline No & $10(27)$ & $27(73)$ & 1.00 & - & - & 1.00 & - & - \\
\hline \multicolumn{9}{|l|}{ Redo surgery } \\
\hline Yes & $0(0)$ & $16(100)$ & 0.07 & $0.00-1.20$ & 0.06 & 0.85 & $0.67-1.07$ & 0.16 \\
\hline No & $12(31)$ & $27(69)$ & 1.00 & - & - & 1.00 & - & - \\
\hline \multicolumn{9}{|l|}{ Extent of resection } \\
\hline GTR & $9(22)$ & $32(78)$ & 1.03 & $0.24-4.51$ & 0.97 & 1.04 & $0.83-1.32$ & 0.70 \\
\hline STR/biopsy & $3(21)$ & $11(79)$ & 1.00 & - & - & 1.00 & - & - \\
\hline \multicolumn{9}{|l|}{ Surgical exposure } \\
\hline Craniotomy & $12(27)$ & $32(73)$ & 8.85 & $0.48-161.68$ & 0.14 & 0.95 & $0.73-1.24$ & 0.71 \\
\hline Craniectomy & $0(0)$ & $11(100)$ & 1.00 & - & - & 1.00 & - & - \\
\hline \multicolumn{9}{|l|}{ Surgical approach* } \\
\hline Transvermian & $10(42)$ & $14(58)$ & 1.00 & - & - & 1.00 & - & - \\
\hline Telovelar & $2(8)$ & $24(92)$ & 8.57 & $1.64-44.86$ & 0.01 & 0.82 & $0.71-0.95$ & 0.01 \\
\hline Other & $0(0)$ & $4(100)$ & 6.52 & $0.31-134.60$ & 0.22 & - & - & - \\
\hline \multicolumn{9}{|l|}{ Surgical adjuncts } \\
\hline \multicolumn{9}{|l|}{ Intraop EVD* } \\
\hline Yes & $9(64)$ & $5(36)$ & 0.04 & $0.01-0.22$ & $<0.001$ & 1.47 & $1.17-1.83$ & $<0.001$ \\
\hline No & $3(7)$ & $38(93)$ & 1.00 & - & - & 1.00 & - & - \\
\hline
\end{tabular}

Data are presented as number of patients (\%). Boldface type indicates statistical significance.

${ }^{*} p \leq 0.05$.

superior cerebellar peduncles are particularly vulnerable, and over-manipulation of these structures can cause disturbances of equilibrium associated with truncal ataxia and staggering gait. ${ }^{20,22}$ If the dentate nuclei, which lie rostral to the superior pole of the tonsils, are damaged, significant disturbance of equilibrium can occur. ${ }^{20}$

Postoperative gait disturbance is described in the literature. ${ }^{67,19}$ In a clinical series (15 patients) describing the surgical outcome of large fourth ventricle tumors in children, Rajesh et al.$^{19}$ reported immediate postoperative ataxia in $47 \%$ of patients, which is similar to our result. Splitting of the vermis for fourth ventricle access has been frequently scrutinized in the literature because it requires incision into functional areas of the cerebellum. In theory, the transvermian approach may compromise cerebellar projections associated with the vestibular system, and their interruption can cause disturbance of equilibrium and resultant ataxia. ${ }^{20,22}$ Accordingly, our multivariate analysis showed that patients undergoing the telovelar approach were in fact significantly less likely to experience postoperative gait disturbance.

\section{Speech and Swallowing Function}

One-third of patients had postoperative speech and/ 
or swallowing deficits. In a number of these patients the impairment was debilitating, resulting in gastrostomy or tracheostomy placement. Speech and swallowing difficulties are among the most frequently discussed complications following posterior fossa surgery. Even though most such series do not focus on fourth ventricle tumors, the same risks and pitfalls appear to apply. These deficits are often described as a component of the "posterior fossa syndrome," which involves a constellation of symptoms, such as mutism, dysarthria, and bulbar symptoms, including dysphagia. ${ }^{10}$ The anatomical substrate of the development of postoperative speech/swallowing difficulties is not completely understood. Oral apraxia is thought to result from impaired coordination of oropharyngeal musculature. Disruption of the dentatothalamocortical tracts, which originate in the dentate nucleus and terminate in cortical premotor and supplementary motor areas, has been heavily implicated. ${ }^{10,16}$ Incision of the vermis (especially the rostral vermis) during the surgical approach is also commonly associated with the development of postoperative speech/swallowing deficits. Midline cerebellar structures have complex connections with cerebellar nuclei, which in turn have connections with the pontine nuclei and thalamus. In addition to receiving tactile information from the limbs, head, and face, the vermis also receives information from cortical areas responsible for speech initiation. Damage to the vermis or paravermian areas may inhibit oropharyngeal motor control via interruption of cerebellar cortical motor input. ${ }^{1,3}$ The telovelar approach has been well described as a way to spare these critical midline structures and avoid this potentially devastating complication. ${ }^{4,17}$

The impact of surgical approach on the development of postoperative speech and swallowing deficits is mixed in the literature. Zaheer and Wood ${ }^{25}$ reported their experience with large pediatric fourth ventricle tumors using the telovelar approach exclusively (20 patients). Even with sparing of the vermis, the authors reported that $30 \%$ of patients developed posterior fossa syndrome. The authors noted that this finding may undermine the role of the vermis in the development of this syndrome. On the other hand, El-Bahy ${ }^{7}$ reported no occurrences of cerebellar mutism in a series of 16 cases in which only the telovelar approach was used. Pollack et al. ${ }^{18}$ reviewed their 9-year experience with posterior fossa tumors (142 patients) and failed to find a correlation between length of vermian incision and the development of speech deficits. In our series, patients undergoing a transvermian approach had significantly more speech/swallowing complications than those undergoing a telovelar approach (54\% vs $31 \%$ ), and this finding persisted in the multivariate analysis. Fortunately, speech/swallowing deficits were the most likely to resolve, with $50 \%$ and $80 \%$ of patients for whom data were available reporting resolution within 30 and 90 days, respectively, and $100 \%(\mathrm{n}=11)$ at 1 year of follow-up.

The other factor we found to be significantly associated with speech/swallowing deficits was anterior tumor extension (tumor abutting or invading the brainstem). This finding is in agreement with previous reports. Doxey et al. ${ }^{6}$ reviewed the surgical outcome for 253 children who underwent resection of posterior fossa tumors complicated by symptoms of posterior fossa syndrome. The authors defined mutism as the inability to articulate single monosyllabic words. All patients who experienced postoperative mutism had tumors that invaded the brainstem or middle cerebellar peduncle.

\section{Cranial Nerves}

Over $30 \%$ of patients had worsening of a cranial nerve deficit or developed a new one. Of the postoperative neurological complications, cranial nerve deficits were the least likely to recover, with less than $10 \%$ of patients returning to their neurological baseline by 90 -day follow-up. Others have reported the occurrence of cranial nerve palsies in small descriptive series. ${ }^{6,19}$ In our series the most common cranial nerves affected were the abducens and facial nerves (CN VI and VII), and this is consistent with the literature. ${ }^{6,15,18}$ Like the majority of cranial nerve palsies associated with fourth ventricle surgery, CN VI and VII deficits are typically caused by manipulation or damage to the ventricle floor along the facial colliculus where the facial nerve loops around the nucleus of CN VI.,15

The only surgical factor significantly associated with cranial nerve deficit development in the multivariate analysis was the transvermian approach. Nineteen percent of patients who underwent a telovelar approach had new or worsening cranial nerve deficits compared with $50 \%$ of those who underwent a transvermian approach. Most of the cranial nerve nuclei are located in close proximity to the median eminence, hence it is possible that this direct midline approach may put these nuclei at slightly increased risk. Additionally, with the transvermian approach, the normal anatomy is not often seen until the end of tumor resection, which may place the fourth ventricle floor at higher risk. Even though the difference was only at the level of a trend, we found that the transvermian approach was more likely to be used with recurrent tumor/ repeat operations. Repeat operations in this region are understandably difficult, with scarring and distorted anatomy as impacting factors. The elevated surgical complexity of repeat operations may contribute to the increased cranial nerve deficits seen with the transvermian approach. The 5\% incidence of new postoperative cranial neuropathy in our telovelar group mirrors that reported (4.5\%) for the largest published series of patients with fourth ventricle tumors treated exclusively by the telovelar approach. ${ }^{23}$ The use of neuromonitoring has been advocated in the literature to avoid damage to cranial nerve nuclei or tracts, ${ }^{11}$ but its use was not associated with better or worse cranial nerve outcome in our series. When involvement of (or adherence to) the brainstem by tumor was suspected, we did use intraoperative neurophysiological monitoring in an effort to enhance safety. We cannot analyze the efficacy of such monitoring in this study, as it was used in a minority of cases, the patients in whom it was used tended to have more difficult, invasive tumors, and its use varied among surgeons.

\section{Postoperative CSF Diversion}

The intermittent necessity of postoperative CSF shunting following tumor resection has been described in the literature. Zaheer and $\mathrm{Wood}^{25}$ reported a postoperative 
shunting rate of $30 \%$ following fourth ventricle tumor resection in pediatric patients. In a report of the surgical outcome for 26 patients with fourth ventricle ependymomas, the authors reported a shunting rate of $23 \% .{ }^{21}$ In our series 12 patients (22\%) had hydrocephalus requiring CSF diversion following tumor resection, and the majority of these patients were children. It was not unexpected that patients who required an intraoperative EVD would be more likely to eventually require permanent shunt placement. Our study shows that two-thirds of patients receiving intraoperative EVDs required postoperative CSF diversion. Interestingly, we found that the telovelar approach resulted in a lower rate of postoperative CSF diversion. This is the first study to report this outcome, which may be due to increased postoperative scar formation in the transvermian approach leading to compromised CSF flow. Following the anatomical planes, as in the telovelar approach, may reduce such scarring. Additionally, in the telovelar approach the foramina of Luschka and Magendie are opened widely, which might improve CSF circulation. Our rate of shunting of $8 \%$ in the telovelar group is in the same range as the $4.5 \%$ reported by Tomasello et al. ${ }^{23}$ for the same approach.

\section{Medical Complications}

Consistent with expectations, medical complications were minimal in this patient cohort in that only a very few patients suffered from systemic metastatic disease. Moreover, the population was relatively young, with an average age of less than 40 years, and younger age is generally correlated with better surgical outcomes. Respiratory complications were notably the most frequent among the medical complications, likely due to postoperative speech/ swallowing deficits from oral apraxia or lower cranial nerve deficits. These deficits can be devastating, especially in combination with facial paresis from CN VII dysfunction, and can result in aspiration pneumonia, leading to prolonged intubation or tracheostomy placement.

\section{Technical Aspects of the Telovelar and Transvermian Approaches}

The telovelar approach was first described by Matsushima et al., ${ }^{13}$ who suggested using the cerebellomedullary fissure to gain access to the fourth ventricle without splitting the vermis. The anatomy was further clarified by Rhoton, ${ }^{20}$ and the 3 parts of the dissection as outlined by Mussi and Rhoton ${ }^{17}$ are: 1) opening the tela choroidea, which exposes the fourth ventricle floor; 2) incising the superior medullary velum, which exposes the ipsilateral superolateral recess; and 3) cutting the tela between tonsil and medulla, which exposes the most caudal portion of the lateral recess. The lateral reach of this approach can be extended by resecting the cerebellar tonsil or by opening both fissures if exposure of both lateral recesses is desired. ${ }^{12}$ In one series, however, a significantly higher rate of mutism was noted in patients undergoing bilateral exposure. ${ }^{8}$ Jittapiromsak et al. ${ }^{9}$ found in cadaveric anatomical preparations that tonsillar resection provided a wider corridor of access to the aqueduct of Sylvius and lateral recess than tonsillar retraction; however, while using the telovelar method we obtained adequate access with retraction alone, perhaps because the flexibility of the brain is greater in living patients than in the formalin-fixed state. In our series of patients we did not remove the tonsil, and all our approaches were unilateral. Opening the inferior medullary velum between the nodule and flocculus can improve visualization of the superior portion of the lateral recess and thus of the aqueduct, while reaching the foramen of Luschka requires extending the opening to the uvulotonsillar fissure. ${ }^{11}$ Although occasionally opening the inferior medullary velum was required for optimal exposure of the superior aspect of the tumor in our series, we prefer to elevate the velum rather than open it. The necessary extent of telovelar opening thus varies from patient to patient and depends on tumor size and extension.

Recognition and preservation of the posterior inferior cerebellar artery (PICA) is important in the telovelar approach. After originating from the vertebral artery, curving up and around the medulla, and going along the midline between the tonsils, it runs between the tonsil's upper pole and the telovelar membrane. ${ }^{17,24}$ It is therefore exposed and at risk during the separation of that membrane during a telovelar approach. With careful microsurgical technique it should be possible to keep the PICA intact; in our series no postoperative infarcts occurred. The other vascular structure encountered during the approach is the vein of the cerebellomedullary fissure, which lies on the telovelar membrane in the fissure and drains to the superior petrosal sinus. It usually is divided during the approach, and its absence leaves no clinical sequelae due to the anastomotic venous network present in this area.

The feasibility of using endoscopy to perform a telovelar approach has been addressed by a report that addresses the endoscopic topographical anatomy of this region. ${ }^{5}$ Because prior reports of endoscopic access to the fourth ventricle used the foramen of Magendie to enter that space, telovelar access by endoscopy has (in theory) the double advantage of minimizing tonsillar retraction and associated injury to the dentate nuclei, while also reducing the degree of telovelar opening required for visualization within the ventricle. 5,14 This method has not been used clinically but shows that minimally invasive surgery may become useful in treating tumors of the fourth ventricle.

The transvermian approach is achieved by incising the inferior vermis and retracting the 2 halves of the vermis laterally to obtain adequate exposure. The length of the vermian incision is not standardized and clearly depends on the size and position of the lesion. Ideally, the incision should be limited as much as feasible to avoid neurological comprise. Unfortunately, this often results is a very small operative window. Additionally, with this approach the surgeon often does not see normal anatomical landmarks until the end of the resection, and tumor manipulation may thus indirectly or inadvertently cause traction or contusive injury to structures in the ventricle floor. Nevertheless, the transvermian approach may prove beneficial in cases where the lesion is in located in the superior half of the fourth ventricle. The required vertical angle, which can sometimes be extreme in the setting of a steep tentorium, may be difficult to achieve with a telovelar approach resulting in a potentially awkward working angle.,22 This is particularly true when the patient is in the prone position. 
In this situation, the more direct transvermian approach may prove useful. Another advantage to the transvermian approach is that it involves significantly less manipulation of the major blood vessels. Lastly, in cases of recurrent lesions with scarring and distortion of the natural planes and corridors, the transvermian approach may provide better and reliable access.

\section{Limitations}

This study has several limitations that warrant discussion, among them its retrospective nature. Even though this is the largest retrospective surgical series of fourth ventricle tumors in the literature, the number of patients is still small, and this affects the power of subgroup analysis. There is probably crossover between the speech/swallowing deficits and cranial nerve deficits. The specific criteria used by each individual surgeon in choosing the surgical approach are unknown. Hence, we cannot eliminate the possibility that the transvermian approach was selected for more complex lesions, which would account for the increased incidence of neurological morbidity in our analysis. The data regarding deficits in speech, swallowing, and other cranial nerve functions were collected from medical records, including otolaryngology, speech pathology, and ophthalmology notes. On clinical evaluation it may be difficult to discern an oral apraxia of pure vermian origin versus lower cranial nerve deficits, especially if such deficits are subtle. Ocular motor defects and facial palsies are much easier to document accurately based solely on clinical examination. Furthermore, although we were able to report complete resolution of deficits in some cases, this does not fully capture clinical improvement. It is likely that in patient records, subtle, gradual improvement in deficits may be less meticulously documented than complete resolution. This may be true of subtle cranial nerve deficits, which may explain why their reported rate of resolution is lower. Ideally, a specific scale that more effectively quantifies the severity of reported cranial nerve deficits would better capture patient improvement; however, in this regard we are limited by the retrospective nature of the data collection.

\section{Conclusions}

Fourth ventricle tumors represent a difficult clinical challenge. Our analysis focused on surgical complications, which are poorly reported. Studies with longer follow-up periods are certainly needed to assess the lifetime impact of surgery. However, this report demonstrates that the neurological sequelae of fourth ventricle surgery are not insignificant. The occurrence of cranial nerve deficits may be higher than previously reported, and they are less likely to resolve than gait or speech/swallowing deficits. Additionally, our report indicates that the telovelar approach is associated with less neurological morbidity and lower rates of shunting after tumor removal compared with the transvermian approach.

\section{References}

1. Carpenter MB: Core Text of Neuroanatomy. Baltimore: Williams \& Wilkins, 1985
2. Cohen AR (ed): Surgical Disorders of the Fourth Ventricle. Cambridge, MA: Blackwell Science, 1996, pp 147-160

3. Dailey AT, McKhann GM II, Berger MS: The pathophysiology of oral pharyngeal apraxia and mutism following posterior fossa tumor resection in children. J Neurosurg 83:467-475, 1995

4. Deshmukh VR, Figueiredo EG, Deshmukh P, Crawford NR, Preul MC, Spetzler RF: Quantification and comparison of telovelar and transvermian approaches to the fourth ventricle. Neurosurgery 58(4 Suppl 2):ONS-202-ONS- 207, 2006

5. Di Ieva A, Komatsu M, Komatsu F, Tschabitscher M: Endoscopic telovelar approach to the fourth ventricle: anatomic study. Neurosurg Rev 35:341-349, 2012

6. Doxey D, Bruce D, Sklar F, Swift D, Shapiro K: Posterior fossa syndrome: identifiable risk factors and irreversible complications. Pediatr Neurosurg 31:131-136, 1999

7. El-Bahy K: Telovelar approach to the fourth ventricle: operative findings and results in 16 cases. Acta Neurochir (Wien) 147:137-142, 2005

8. Han S, Wang Z, Wang Y, Wu A: Transcerebellomedullary fissure approach to lesions of the fourth ventricle: less is more? Acta Neurochir (Wien) 155:1011-1016, 2013

9. Jittapiromsak P, Sabuncuoglu H, Deshmukh P, Spetzler RF, Preul MC: Accessing the recesses of the fourth ventricle: comparison of tonsillar retraction and resection in the telovelar approach. Neurosurgery 66 (3 Suppl Operative):30-40, 2010

10. Korah MP, Esiashvili N, Mazewski CM, Hudgins RJ, Tighiouart M, Janss AJ, et al: Incidence, risks, and sequelae of posterior fossa syndrome in pediatric medulloblastoma. Int J Radiat Oncol Biol Phys 77:106-112, 2010

11. Lee CC, Lin CF, Yang TF, Hsu SP, Chen HH, Chen SC, et al: Telovelar approach for choroid plexus papilloma in the foramen of Luschka: a safe way using a neuromonitor. Clin Neurol Neurosurg 114:249-253, 2012

12. Liu R, Kasper EM: Bilateral telovelar approach: A safe route revisited for resections of various large fourth ventricle tumors. Surg Neurol Int 5:16, 2014

13. Matsushima T, Fukui M, Inoue T, Natori Y, Baba T, Fujii K: Microsurgical and magnetic resonance imaging anatomy of the cerebello-medullary fissure and its application during fourth ventricle surgery. Neurosurgery 30:325-330, 1992

14. Matula C, Reinprecht A, Roessler K, Tschabitscher M, Koos WT: Endoscopic exploration of the IVth ventricle. Minim Invasive Neurosurg 39:86-92, 1996

15. Miller J, Hdeib A, Cohen A: Management of tumors of the fourth ventricle, in Quiñones-Hinojosa A (ed): Schmidek and Sweet: Operative Neurosurgical Techniques, ed 6. Philadelphia: Saunders Elsevier, 2012, Vol 1, pp 367-398

16. Morris EB, Phillips NS, Laningham FH, Patay Z, Gajjar A, Wallace D, et al: Proximal dentatothalamocortical tract involvement in posterior fossa syndrome. Brain 132:30873095, 2009

17. Mussi AC, Rhoton AL Jr: Telovelar approach to the fourth ventricle: microsurgical anatomy. J Neurosurg 92:812-823, 2000

18. Pollack IF, Polinko P, Albright AL, Towbin R, Fitz C: Mutism and pseudobulbar symptoms after resection of posterior fossa tumors in children: incidence and pathophysiology. Neurosurgery 37:885-893, 1995

19. Rajesh BJ, Rao BR, Menon G, Abraham M, Easwer HV, Nair S: Telovelar approach: technical issues for large fourth ventricle tumors. Childs Nerv Syst 23:555-558, 2007

20. Rhoton AL Jr: Cerebellum and fourth ventricle. Neurosurgery 47 (3 Suppl):S7-S27, 2000

21. Spagnoli D, Tomei G, Ceccarelli G, Grimoldi N, Lanterna A, Bello L, et al: Combined treatment of fourth ventricle ependymomas: report of 26 cases. Surg Neurol 54:19-26, 2000

22. Tanriover N, Ulm AJ, Rhoton AL Jr, Yasuda A: Comparison 
of the transvermian and telovelar approaches to the fourth ventricle. J Neurosurg 101:484-498, 2004

23. Tomasello F, Conti A, Cardali S, La Torre D, Angileri FF: Telovelar approach to fourth ventricle tumors: highlights and limitations. World Neurosurg 83:1141-1147, 2015

24. Ucerler H, Saylam C, Cagli S, Orhan M, Zileli M: The posterior inferior cerebellar artery and its branches in relation to the cerebellomedullary fissure. Clin Anat 21:119-126, 2008

25. Zaheer SN, Wood M: Experiences with the telovelar approach to fourth ventricular tumors in children. Pediatr Neurosurg 46:340-343, 2010

\section{Disclosures}

The authors report no conflict of interest concerning the materials or methods used in this study or the findings specified in this paper.

\section{Author Contributions}

Conception and design: Ferguson, Levine, Tsung. Acquisition of data: Ferguson. Analysis and interpretation of data: Ferguson, Levine, Suki, McCutcheon. Drafting the article: Ferguson, Suki, Tsung, Lang, Weinberg, McCutcheon. Critically revising the article: all authors. Reviewed submitted version of manuscript: Ferguson, Levine, Tsung, Lang, Sawaya, Weinberg, McCutcheon. Approved the final version of the manuscript on behalf of all authors: Ferguson. Statistical analysis: Ferguson, Suki, Tsung. Administrative/technical/material support: Suki. Study supervision: Levine, Lang, McCutcheon.

\section{Correspondence}

Sherise D. Ferguson, Department of Neurosurgery, MD Anderson Cancer Center, 1400 Holcombe Blvd., Houston, TX 77030. email: sdferguson@mdanderson.org. 